\title{
Evaluation of Fluid-Thermal Systems by Dynamic Data Driven Application Systems
}

\author{
D. Knight, T. Rossman, and Y. Jaluria \\ Dept of Mechanical and Aerospace Engineering \\ Rutgers - The State University of New Jersey \\ New Brunswick, NJ 08903 \\ knight@soemail.rutgers.edu
}

\begin{abstract}
A Dynamic Data Driven Application Systems (DDDAS) approach is developed for evaluation of fluid-thermal systems wherein a complete specification of the boundary conditions is not known a priori and experimental diagnostics are restricted to a limited region of the flowfield. The methodology is applied to the configuration of a heated jet injected into a laminar boundary layer where the jet temperature is not known a priori. Preliminary results are presented.
\end{abstract}

\section{Introduction}

The design and control of efficient fluid-thermal systems requires detailed quantitative information on the flowfield (e.g., pressure, species concentration, temperature and velocity). Examples of such systems are combustors, furnaces, and reactors [1. In many situations, very limited access to the flow domain is available and strong constraints are thus placed on the experimental data that can be obtained through non-intrusive (e.g., optical) and intrusive techniques. An example is the optical fiber drawing furnace, which typically has an infrared sensor to monitor the temperature of the heating element at only one location and must use this single source of information to control the optical fibre drawing process [2, 3, 4. Another example is the combustor in a turbofan engine.

A common approach is to use numerical simulation (i.e., Computational Fluid Dynamics $[\mathrm{CFD}]$ ) to obtain the desired information in the given domain using the available experimental data. However, this procedure can be successful only if the boundary conditions for the numerical simulation are well defined. In many cases of practical interest, such as the draw furnace and combustion chamber mentioned previously, the boundary conditions (in particular, the thermal conditions) are not known with sufficient accuracy or even at all. In the optical fibre drawing furnace, for example, the temperature distribution at the wall is the result of the overall heat transfer processes in the domain and cannot be taken as a known input to the model. This temperature distribution affects the fluid properties, as well as chemical reactions and the transport mechanisms in the flow. The wall temperature distribution is critical to the calculation of the glass flow and thus the characteristics of the fiber. However, it is typically infeasible to measure the wall temperature, and hence the information regarding the 
boundary conditions is incomplete. It is possible to infer the boundary conditions from an inverse procedure. For example, Issa et al [2] determined the temperature distribution at the wall of a cylindrical fiber drawing furnace by using the limited experimental data obtained by measuring the temperature at the axis of a graphite rod located at the center of the furnace using an inverse calculation based on the governing transport equations coupled with an optimization procedure.

\section{Dynamic Data Driven Application Systems}

The Dynamic Data Driven Applications Systems (DDDAS) concept was described in the DDDAS Workshop held at the National Science Foundation in March 2000 [5. DDDAS is a novel approach to engineering and scientific research wherein experiment and simulation interact in a synergistic, symbiotic manner. DDDAS can be implemented in either an open loop or closed loop manner. In the former case, experimental data is streamed into the simulation (or vice-versa) to achieve greater accuracy, detail and/or robustness. An example is real time streaming of meteorological measurements into weather simulations. In the latter case, experiment and simulation interact iteratively, where the experiment guides the simulation, and the simulation in turn guides the experiment (see Darema [6]). The recent Dynamic Data Driven Applications Systems Workshop in January 2006 [5] summarized major achievements using the DDDAS approach and identified additional opportunities. Additional information is available on the DDDAS.org homepage [7].

The Dynamic Data Driven Applications Systems concept may be directly applied to significantly improve the evaluation of fluid-thermal systems using combined experiment and simulation. The basic concept is to synergize the experiment and simulation in a manner which exploits the advantages, and recognizes the limitations, of each approach. We specifically focus on systems evaluation in thermal sciences wherein the systems are characterized by two major limitations. First, experimental measurements are limited to optical diagnostics in restricted regions of the flow (e.g., a subsection of the flowfield using optical access along a single direction). Second, there is incomplete a priori specification of the boundary conditions for the flow simulation.

\section{Description of Research}

\subsection{Objective}

The objective of the research is the development of a general methodology for synergizing experiment and simulation for system evaluation in thermal sciences wherein the experimental measurements are restricted in region and scope, and the a priori boundary conditions for simulation are incomplete. The approach is firmly based on the DDDAS concept wherein the experiment directs the simulation and vice-versa in a closed-loop manner. The basic approach is to approximate the unknown boundary conditions by minimizing the error in the prediction 
of the measured data (i.e., the experiment driving the simulation), and to identify needed subsequent experimental measurements to reduce the error (i.e., the simulation driving the experiment).

\subsection{Configuration}

We consider a rectangular jet injected perpendicular to an incompressible laminar boundary layer. The configuration is shown in Fig. 11 The inflow is an equilibrium laminar boundary layer in air defined by the specified freestream conditions (velocity $U_{\infty}$, static pressure $p_{\infty}$ and static temperature $T_{\infty}$ ) and boundary layer thickness 1$] \delta_{\infty}$. The jet is defined by the jet average velocity $U_{j}$, static pressure $p_{j}$, and static temperature $T_{j}$.

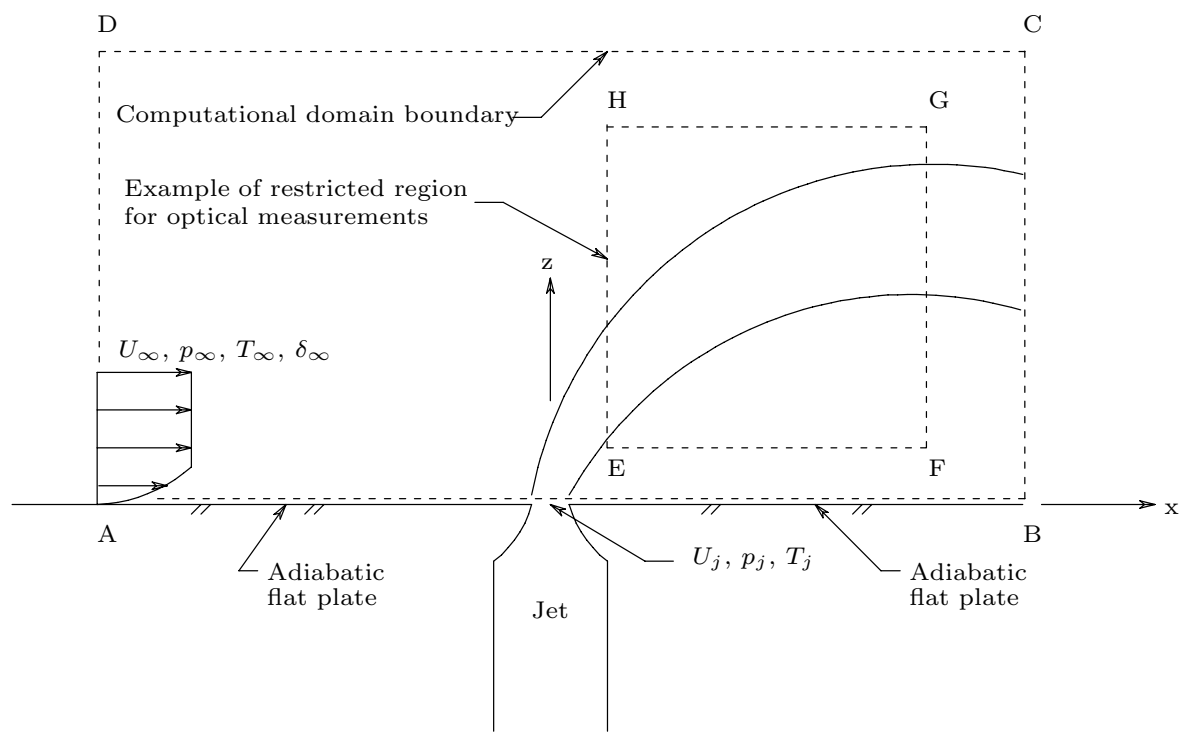

Fig. 1. Flow configuration

The computational domain ABCD is shown in Fig. 1, The inflow conditions $\left(U_{\infty}, p_{\infty}, T_{\infty}\right.$ and $\left.\delta_{\infty}\right)$ are assumed known. By analogy to the optical fiber furnace, the jet exit temperature $T_{j}$ is assumed unknown, while the jet exit pressure $p_{j}$ and average velocity $U_{j}$ are assumed known. In future work, more complex problems will be considered. The specific values are indicated in Table1.

The objective of this initial research problem is the determination of the jet exit temperature $T_{j}$ through a DDDAS methodology using a closed-loop iteration of experiment and simulation. The experimental data are diode laser absorption measurements $8,9,10$. The simulation is performed using the commercial

${ }^{1}$ Here, $\delta_{\infty}$ is the laminar boundary layer thickness that would exist at the location of the jet exit in the absence of the jet. Equivalently, the distance from the leading edge of the flat plate to the jet exit may be specified. 
Table 1. Flow Conditions

\begin{tabular}{lr} 
Quantity & Value \\
\hline \hline$U_{\infty}(\mathrm{m} / \mathrm{s})$ & 5 \\
$p_{\infty}(\mathrm{kPa})$ & 101 \\
$T_{\infty}(\mathrm{K})$ & 298 \\
$U_{j}(\mathrm{~m} / \mathrm{s})$ & 15 \\
$p_{j}(\mathrm{kPa})$ & 101 \\
$T_{j}(\mathrm{~K})$ & $\mathrm{TBD}$ \\
$L(\mathrm{~mm})$ & 158 \\
$W(\mathrm{~mm})$ & 3.2
\end{tabular}

LEGEND

$L \quad$ distance from leading edge to jet

$W \quad$ width of jet

TBD to be determined by DDDAS

Fluent software [1] which solves the incompressible Navier-Stokes equations using a spatially and temporally second-order accuracy finite volume algorithm.

\subsection{DDDAS Methodology}

A DDDAS methodology is used to determine the optimal location of the experimental measurements through an iterative procedure involving experiment and simulation. The methodology is illustrated in Fig. 2. Consider a diode laser absorbance measurement in a vertical plane at a specific location $(x, z)$ within a limited region EFGH shown in Fig. 11. The relative instantaneous absorbance $\mathcal{A}(x, z, t)$ over a spanwise distance $y_{1} \leq y \leq y_{2}$ is defined as

$$
\mathcal{A}(x, z, t)=\frac{\left(I_{o}-I(x, z, t)\right)}{I_{o}}
$$

where $I_{o}$ is the incident intensity at $\left(x, y_{1}, z\right)$ and $I(x, z, t)$ is the instantaneous transmitted intensity at $\left(x, y_{2}, z\right)$. The absorbance $\mathcal{A}(x, z, t)$ can also be accurately calculated from Beer-Lambert's Law

$$
\mathcal{A}(x, z, t)=1-\exp \left[-\int_{y_{1}}^{y_{2}} \kappa_{\nu} d y\right]
$$

assuming the diode laser frequency $\nu$, mean partial pressure $p_{i}(x, y, z, t)$ of the specific absorbing species $\left(e . g ., \mathrm{O}_{2}\right)$ and static temperature $T(x, y, z, t)$ are known. The absorbance per cm of the ${ }^{Q} R_{2}(6)$ line of the oxygen $b_{1} \Sigma_{g}^{+} \nu^{\prime}=0 \leftarrow$ $X^{3} \Sigma_{g}^{-}, \nu^{\prime \prime}=0$ transition at $761.139 \mathrm{~nm}$ is

$$
\frac{d \mathcal{A}}{d y}=0.083 T^{-1}-2.26 \cdot 10^{-5}
$$

where $T(x, y, z, t)$ is the static temperature in K. We identify the experimental measured absorbance in (11) by $\mathcal{A}_{e}(x, z, t)$ and the simulated absorbance in (2) by $\mathcal{A}_{s}(x, z, t)$. 
Since the flowfield is unsteady as discussed below, the experimental and simulated absorbance power spectra $\mathcal{P}_{e}(f ; x, z)$ and $\mathcal{P}_{s}(f ; x, z)$ are compared where $f$ is the frequency. At a given $(x, z)$-location, we may define the $L_{2}$ norm of the difference between the experimental and simulated absorbance power spectra as

$$
\mathcal{E}(x, z)=\int_{0}^{\infty}\left[\mathcal{P}_{e}(f ; x, z)-\mathcal{P}_{s}(f ; x, z)\right]^{2} d f
$$

The integral (4) is computed at the specific multiple $(x, z)$-locations of the experiment. An rms value of the error $E_{r m s}$ is computed as

$$
\mathcal{E}_{r m s}=\frac{1}{n} \sum_{i} \mathcal{E}\left(x_{i}, z_{i}\right)
$$

where $n$ is the number of experimental location measurements.

The error $\mathcal{E}_{r m s}$ is a function of the assumed value for the jet exit temperature $T_{j}$. We determine a revised value for $T_{j}$ by minimizing the error $\mathcal{E}_{r m s}$ using the Sequential Quadratic Programming procedure CFSQP [12] developed at the University of Maryland.

The flowfield is recomputed using the updated $T_{j}$ and then the flowfield is searched to find the $x$-location where the Total Variation of $\partial \overline{\mathcal{A}}_{s}(x, z) / \partial z$ is the largest, where $\overline{\mathcal{A}}_{s}$ is the time-averaged absorbance at $(x, z)$ and the Total Variation is defined by

$$
\operatorname{TV}\left(\overline{\mathcal{A}}_{s}(x)\right)=\int_{z_{1}}^{z_{2}}\left|\frac{\partial \overline{\mathcal{A}}_{s}}{\partial z}\right| d z
$$

This heuristic algorithm is based on the concept that the optimal $x$-plane location for the next experimental measurement is characterized by the largest variations in the experimental measurement over the $x$-plane.

\section{Results}

We present the preliminary results of our DDDAS methodology (Fig. 2) for evaluation of the fluid-thermal system shown in Fig. 1 based upon the first four months of our research project. A time-accurate Navier-Stokes simulation was performed for the flow conditions shown in Table 1 using an initial jet temperature $T_{j}=398 \mathrm{~K}$. Instantaneous temperature contour.2 3 are shown in Figs. 3 to 6 at intervals 3 of $40 \mathrm{~ms}$. The flowfield is strongly unsteady. The hot jet fluid is entrained into large spanwise vortices which convect downstream. Since the absorbance is inversely proportional to temperature (see (3)), the instantaneous absorbance signal at a fixed location $(x, z)$ varies as each vortex is convected past.

2 The axes are in meters.

${ }^{3}$ For comparison, the time required for the freestream flow to traverse from the left to right boundaries of the computational domain is $155 \mathrm{~ms}$. 


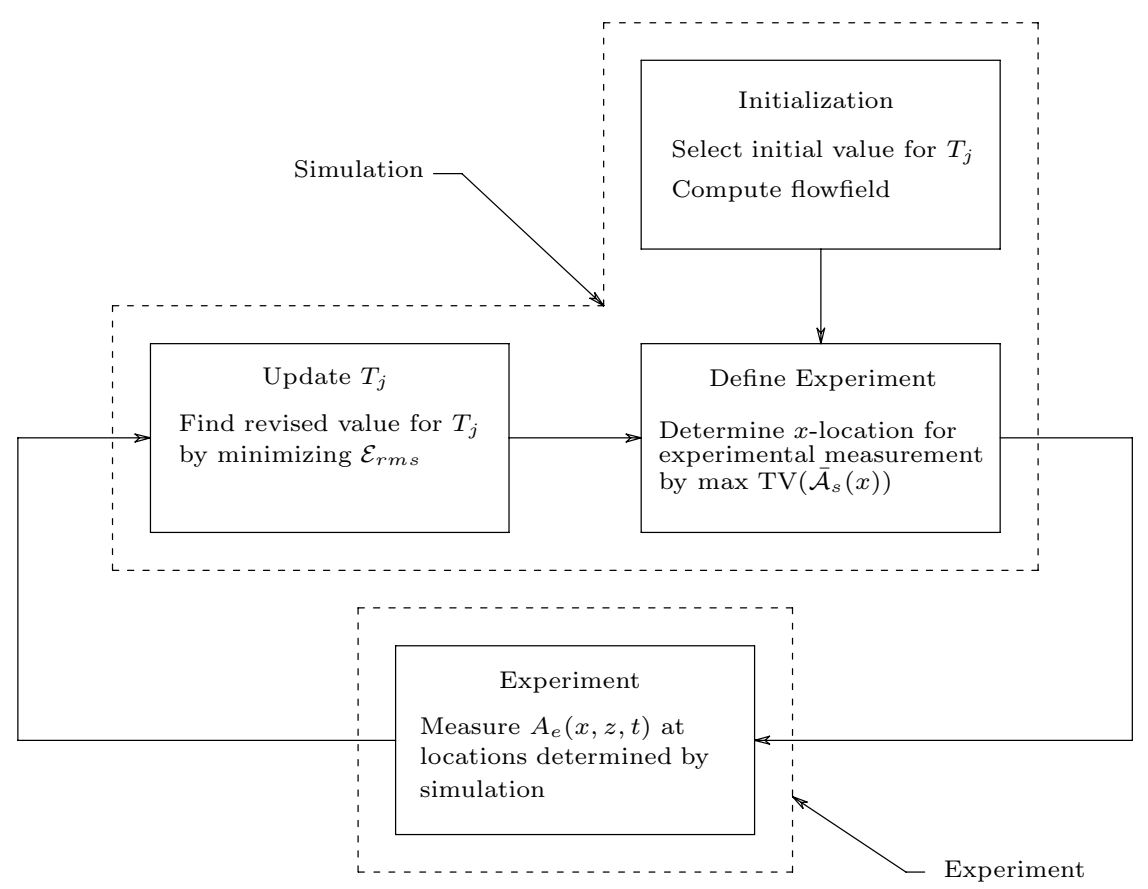

Fig. 2. DDDAS Methodology

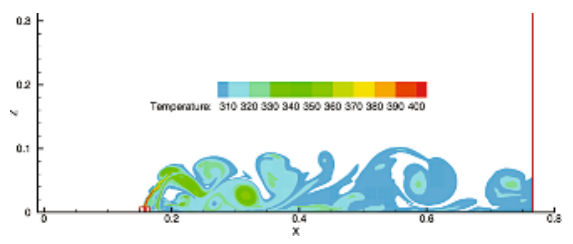

Fig. 3. $t=0$

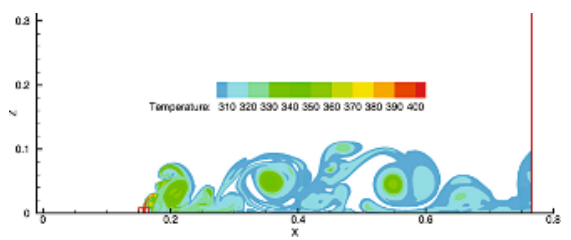

Fig. 5. $t=80 \mathrm{~ms}$

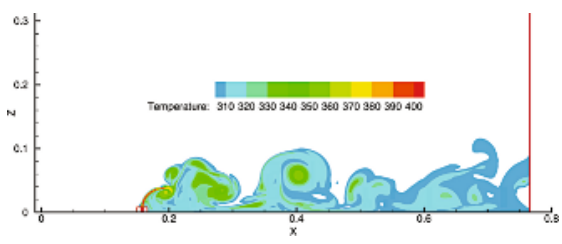

Fig. 4. $t=40 \mathrm{~ms}$

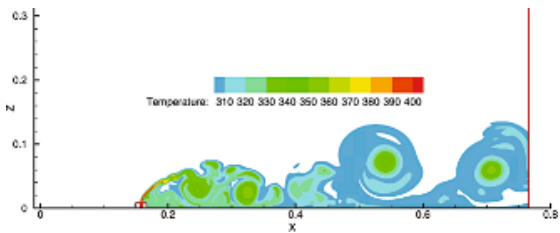

Fig. 6. $t=120 \mathrm{~ms}$

An experimental facility (Fig. 7) has been constructed in the low speed wind tunnel at Rutgers University for the problem configuration (Fig. 1). Initial measurements of oxygen absorption were taken $30 \mathrm{~mm}$ downstream of the jet exit and $30 \mathrm{~mm}$ above the wall based upon the simulation results which indicated that the temperature fluctuations would be large at this location (see Figs. 3 to 
6). The experimental inflow conditions were similar to the simulation; however, the jet velocity $U_{j}=8.7 \mathrm{~m} / \mathrm{s}$. The absorption path was a dual-pass geometry with the laser traversing the flowfield twice at a half angle of 0.2 degrees. Therefore, the effective spatial resolution of the diagnostic is $5 \mathrm{~mm}$. The raw absorbance data traces were reduced to find temperature as a function of time using (3).

The temperature results display a highly unsteady behavior similar to the simulation. The power spectrum of the experimental temperature fluctuations (Fig. 8) shows evidence of vortex shedding behavior with characteristic frequencies at 8 and $15 \mathrm{~Hz}$.

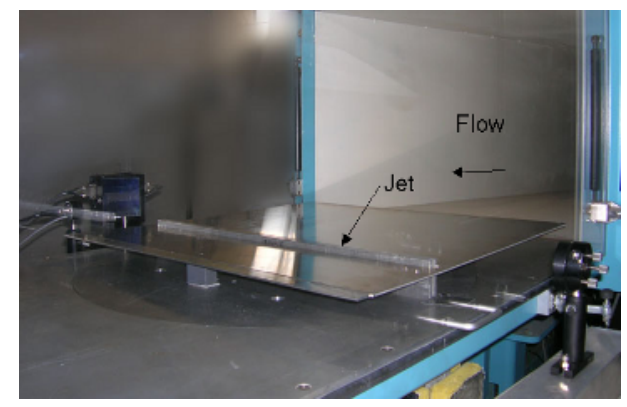

Fig. 7. Experimental configuration

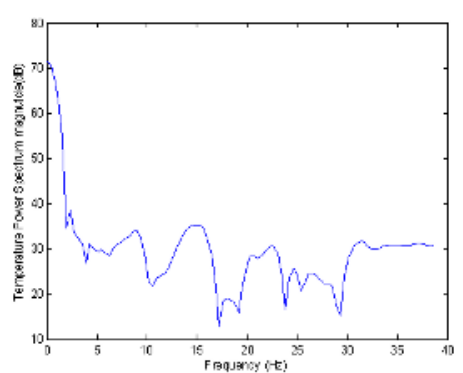

Fig. 8. $\mathcal{P}_{e}$ at $x=30 \mathrm{~mm}, z=30 \mathrm{~mm}$

\section{Future Work}

Our immediate future work will focus on three problems. First, we will complete the determination of the jet temperature $T_{j}$ (and therefore the complete evaluation of the fluid-thermal system in Fig. 1) using our DDDAS methodology. Second and third, we will extend the complexity of the problem to two a priori unknown boundary conditions $\left(T_{j}\right.$ and $\left.U_{j}\right)$ and then three a priori unknown boundary conditions $\left(T_{j}, U_{j}\right.$ and $\left.p_{j}\right)$. This sequence of problems will validate the accuracy and robustness of our DDDAS approach.

\section{Conclusions}

A methodology for evaluation of fluid-thermal systems is developed based upon the Dynamic Data Driven Application Systems approach. The methodology is intended for fluid-thermal systems where complete specification of the boundary conditions is not known a priori and experimental measurements are restricted to a subregion of the fluid-thermal domain. The methodology synergizes experiment and simulation in a closed-loop, iterative manner to achieve a full evaluation of the fluid-thermal system. Preliminary results are presented for the configuration of a heated jet injected into a laminar boundary layer where the jet temperature is not known a priori. Future work is described. 


\section{Acknowledgments}

The research is sponsored by the US National Science Foundation under grant CNS-0539152 (1 Oct 2005 - 30 Sept 06). The program manager is Dr. Frederica Darema.

\section{References}

1. Y. Jaluria. Design and Optimization of Thermal Systems. McGraw-Hill, New York, 1998.

2. J. Issa. Temperature Measurement in an Optical Fiber Draw Furnace. MS Thesis, Dept of Mechanical and Aerospace Engineering, Rutgers University, 1995.

3. S. Roy Choudhury and Y. Jaluria. Practical Aspects in the Drawing of an Optical Fiber. Journal of Materials Research, 13:483-493, 1998.

4. Y. Jaluria. Thermal Processing of Materials: From Basic Research to Engineering. Journal of Heat Transfer, 125:957-979, 2003.

5. Dynamic Data Driven Applications Systems (DDDAS) Website. National Science Foundation, http://www.nsf.gov/cise/cns/darema/dddas/index.jsp.

6. F. Darema. Dynamic Data Driven Applications Systems: A New Paradigm for Application Simulations and Measurements. In Fourth International Conference on Computational Science, pages 662-669, Berlin, 2004. Springer-Verlag.

7. Dynamic Data Driven Applications Systems (DDDAS) Homepage. http://www.dddas.org/.

8. S. Chou, D. Baer, and R. Hanson. Diode-laser Absorption Measurements of $\mathrm{CH}_{3} \mathrm{Cl}$ and $\mathrm{CH}_{4}$ Near $1.65 \mu \mathrm{m} "$. Applied Optics, 36:3288-3293, 1997.

9. R. Mihalcea, D. Baer, and R. Hanson. Diode Laser Sensor for Measurements of $\mathrm{CO}, \mathrm{CO}_{2}$ and $\mathrm{CH}_{4}$ in Combustion Flows. Applied Optics, 36:8745-8752, 1997.

10. D. Nelson, M. Zahniser, J. McManus, C. Kolb, and J. Jimenez. A Tunable Diode Laser System for the Remote Sensing of On-road Vehicle Emissions. Applied Physics B, 67:433-441, 1998.

11. Fluent Version 6. Fluent, Inc. http://www.fluent.com.

12. C. Lawrence, J. Zhou, and A. Tits. Users Guide for CFSQP Version 2.3: A C Code for Solving (Large Scale) Constrained Nonlinear (Minimax) Optimization Problems, Generating Iterates Satisfying All Inequality Constraints. Technical Report TR-94-16r1, Institute for Systems Research, University of Maryland, November 1994. 